\title{
Weather Monitoring System for Renewable Energy Power Production Correlation
}

\author{
Marcos Afonso, Pedro Pereira, and João Martins \\ CTS, Uninova \\ Departamento de Engenharia Electrotécnica \\ Faculdade de Ciências e Tecnologia, FCT, Universidade Nova de Lisboa \\ 2829-516 Caparica, Portugal \\ \{maa18899, pmrp, jf.martins\} @fct.unl.pt
}

\begin{abstract}
This work describes the development of a system designed for renewable power generation integration. It continuously acquires wind, solar and temperature data, which is automatically correlated with energy parameters, obtained from renewable energy systems. The developed system was installed in an urban building equipped with photovoltaic cells and wind renewable generation. To validate the developed application, it was analyzed data of a wind generator and a set of photovoltaic panels, installed near to the weather station. The developed application allows, in addition to the acquisition of weather and energy data, their continuous monitoring and correlation through a graphical user interface, providing a friendly interactivity with the user.
\end{abstract}

Keywords: Weather Conditions, Energy Efficient Buildings, Renewable Energy Production.

\section{Introduction}

The use of fossil fuels - coal, oil and gas - as the primary energy source, was one of the main factors that made possible the rapid Humanity growth in the last century. However, from the oil crisis in the 70's, renewable energies started to have a significant role as a potential alternative to the fossil fuels [1]. The demand and energy consumption in any country is directly connected to its demography and development, having a strong impact on economic growth due to energy prices, particularly prices of fossil fuels [2].

Renewable electric energy production's efficiency depends not only on the existent technology, but also mainly on the weather conditions at a given location. Knowing the weather conditions at a specific place, it is possible to optimize systems that take advantage of renewable energy sources. Moreover, accurate meteorological records are crucial to accurately predict and monitor any energy production system. There are currently a wide range of commercial systems that are able to acquire weather conditions. From basic weather stations, capable of acquiring temperature, humidity and barometric pressure, to the more advanced, which, in addition, are able to measure, and record, wind speed and direction, precipitation and solar radiation. However, these commercial systems only allow the monitoring, and recording, of weather conditions. 
In [3], a non-commercial weather acquisition system for monitoring a photovoltaic (PV) system is proposed. The system is able to acquire solar radiation and temperature, as well as the PV electric parameters, trough a set of sensors connected to a microprocessor, used as the core of the system. The collected data are sent to a computer that works mainly as a database, and the system does not offer a graphical interface. The hardware involved makes the system considerably complex, thus not flexible to changes, making it difficult to adapt to other type of sensors. A similar system is also proposed in [4]. Here, authors stated as major advantages the hardware design and the possibility of operation in remote places, since the system is battery powered. In [5] is described a LabVIEW based system. All data is acquired and processed by LabVIEW, and the user can interact with the system trough some graphical interface menus. However, the tool is only dedicated to PV systems. The system proposed in [6] proposes, as main characteristic, the potential to integrate different renewable energy sources, namely PV and wind generator systems. The core of the system is also a tool developed in LabVIEW. However, the graphical interface and the options presented are limited.

A full system for weather monitoring and renewable energy production correlation could become quite expensive, particularly when small renewable energy systems $(<$ $5 \mathrm{kWp}$ ) are considered. The system costs are the main reason why typically they are only used to: (i) demonstrate that the PV system is a reliable energy source for the given application, or (ii) to develop criteria for design and operation that optimize a PV system for its site and task [7].

In this work, we propose a tool for the management and management of weather data, as well as the correlation among meteorological of energy production data. Meteorological data will be acquired by a weather station equipped with several sensors; all of them installed in the same location as the renewable energy systems. There will be a periodic acquiring of the relevant atmospheric values, including wind speed and direction, temperature and solar radiation. Electrical power generation data will be obtained from another system designed for this purpose and already implemented [8]. With this work, it is our ambition to overcome some of the limitation of the existent systems. The developed tool has three main goals: (i) communication and data acquisition through the weather station; (ii) data processing and (iii) analysis and correlation of meteorological data with data acquired from renewable power generation systems. The developed tool was implemented in Matlab.

\section{Contribution for Sustainability}

Due to the continuous increase in energy consumption, connected to the fact that fossil fuels are becoming more rare and more expensive over the years, the market of renewable energy production systems has increased, mainly in the residential and small-medium enterprises sectors. The developed system, here presented, has two major goals: (1) to offer the possibility of estimating the energy production of new systems to be installed, and (2) to monitoring an existent renewable energy production system, correlating weather and energy data. This system aims to support and validate new technologies applied to renewable energy production systems, since "green" sources play an important role in today's electric grid sustainability. 


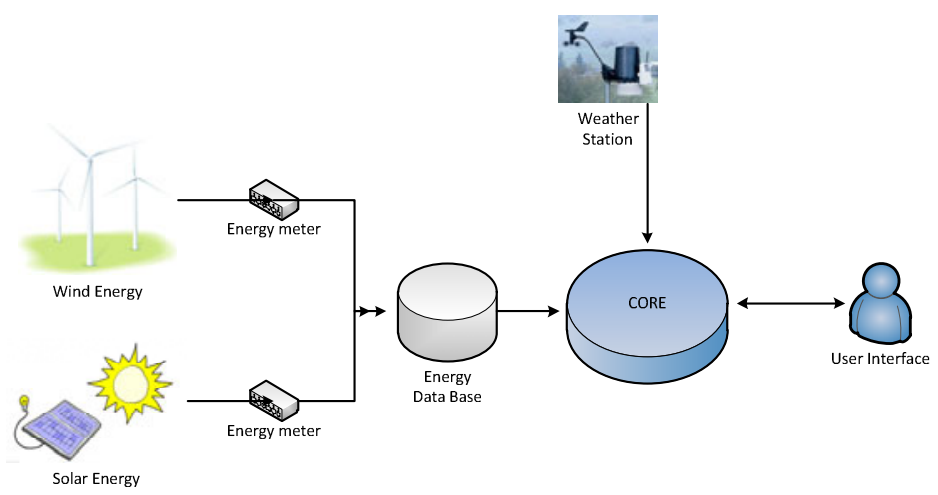

Fig. 1. Conceptual model

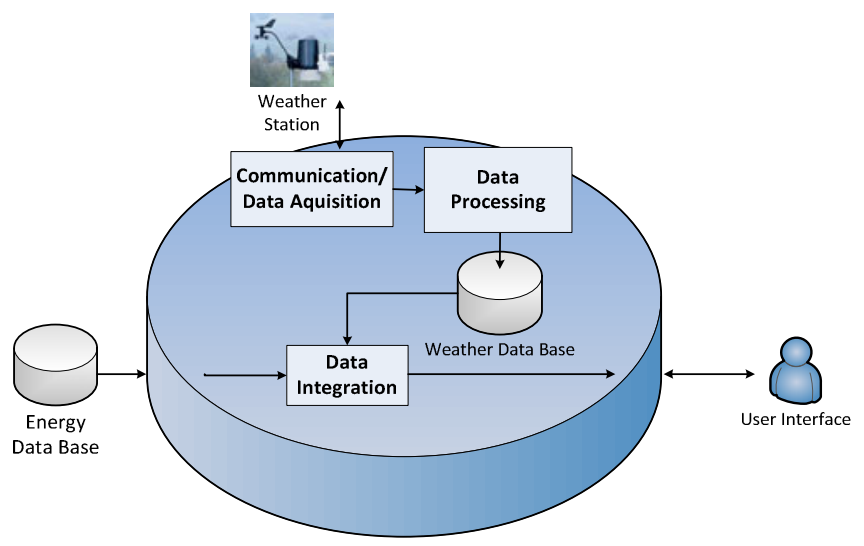

Fig. 2. Core block tasks

The developed system integrates two major areas of interest: (1) meteorological data and renewable electricity generation. Figure 1 presents the framework of the systems' conceptual model.

In Fig. 1, the "core" block is responsible for connecting the energy database with the weather station and the user. The energy database contains data daily acquired from energy meters connected to the renewable energy production systems - wind and photovoltaic generators. This block acts as the system brain, being responsible for acquiring the meteorological data. Moreover, it correlates the data acquired from the weather station with data acquired from the energy database, as presented in Fig. 2. The system offers a friendly user interface that allows a substantial control on parameterization and visualization data.

\section{Monitoring and Correlation}

The methodology adopted in this work aims to make possible the integration of future add-ons. To accomplish this purpose, the core block is divided in sub-blocks that 
represent essential functions of the system. Those sub-blocks, presented in Fig. 3, will be briefly presented.

\section{Communication/data acquisition}

This block is responsible for the communication with the system hardware (datalogger). Once established the communication, it is possible to configure, in the weather station, several options, such as the reading interval, configure data or activate output ports. Furthermore, the nature of the data acquisition can be also configurated: the user can choose either to acquire data stored in the weather station memory or acquire real time data.

\section{Data processing}

In the data processing block, the data collected by the aforementioned routines is decoded. The processed data is then exported to MS Excel files (one file per day), which are stored in an internal database of the computer where the application is running.

\section{Data integration}

The renewable energy production is monitored by a system [8] that keeps daily records of wind generator and PV system energy data. This data integration block, depending on the available data in the databases, allows the user to choose the day and/or the date range that the he desires to view/correlate.

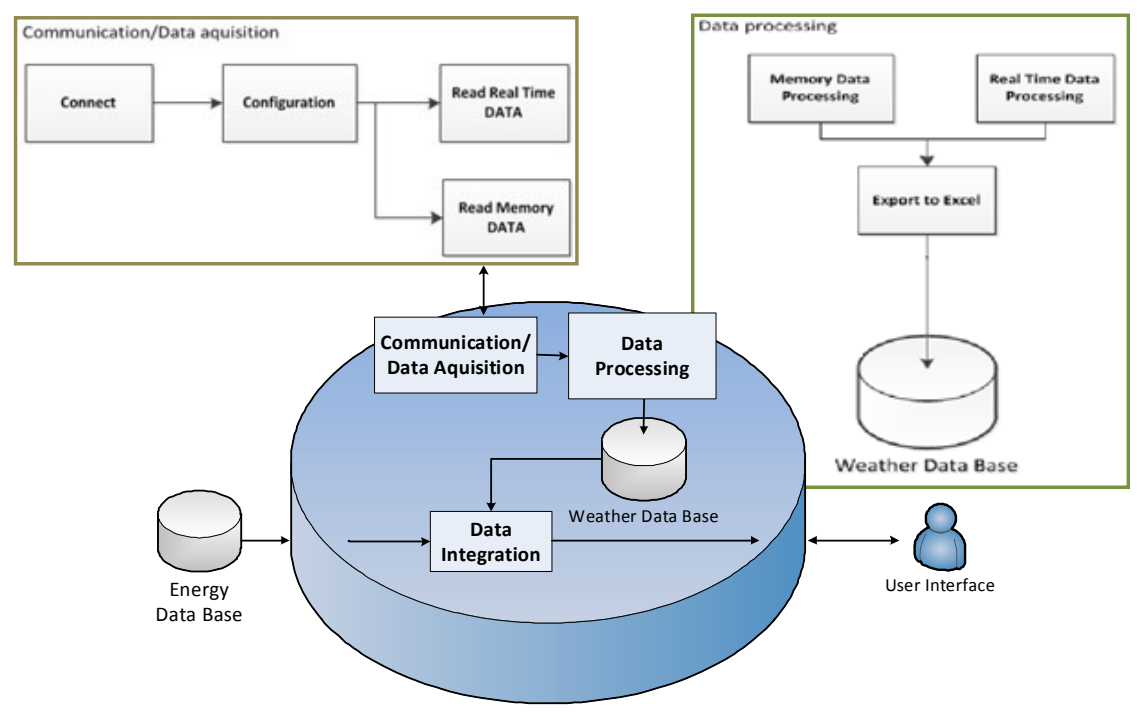

Fig. 3. "Core" sub-blocks

Table 1 presents the several parameters that are suitable for display and correlation. The developed system allows correlation analysis of these parameters (in two different graphics), and also presents a compass rose regarding the set of days selected, correlating wind speed and direction. 
Table 1. List of parameters available for visualization and/or correlation

\begin{tabular}{ll}
\hline $\begin{array}{c}\text { Electrical Energy Production } \\
\text { Parameters (wind and PV) }\end{array}$ & \multicolumn{1}{c}{ Weather Parameters } \\
\hline Voltage (V) & Wind direction $\left({ }^{\circ}\right)$ \\
Current (A) & Wind Gusts $(\mathrm{m} / \mathrm{s})$ \\
Power Factor & Average Wind Speed $(\mathrm{m} / \mathrm{s})$ \\
Power (VA) & Temperature $\left({ }^{\circ} \mathrm{C}\right)$ \\
Active Power (W) & Solar Radiation $\left(\mathrm{W} / \mathrm{m}^{2}\right)$ \\
Reactive Power (VAr) & \\
\hline
\end{tabular}

\section{User Interface}

The user interface is one of the key points of the developed application, providing a friendly and appealing environment to the user. The main idea was to develop an interface that allows the user to have total control over all the involved parameters, either regarding hardware configuration or data acquisition. Figure 4 presents the main window of the implemented tool.

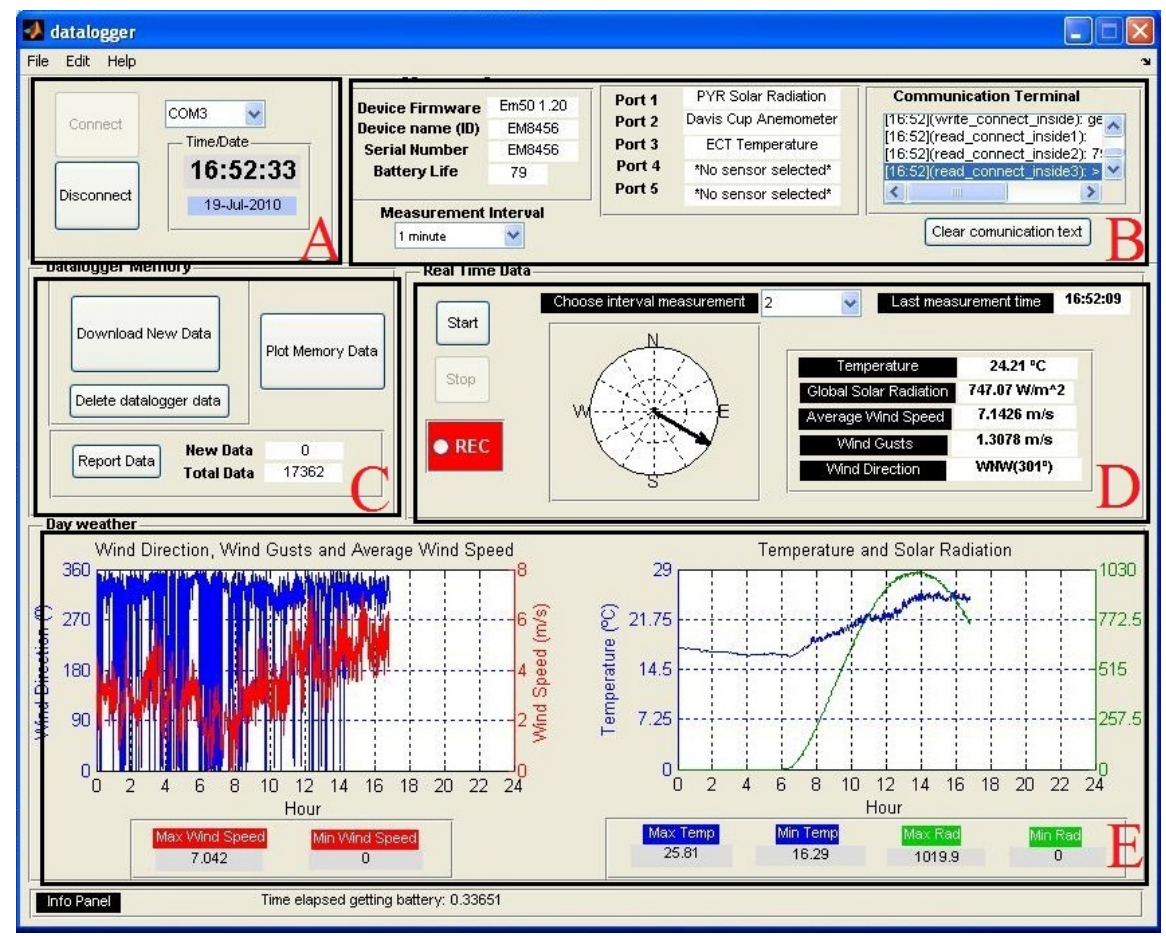

Fig. 4. Main window

In Fig. 4, the main interface is divided um several areas, each one related to a specific task. A summary of each block, identified with capital letters, is presented below. 
- A - Datalogger Control - Starts and stops the connection with the weather station datalogger and gives information about current date and time;

- B - Datalogger Settings - Gives information about the weather station parameters: firmware version number of the device (Device Firmware), device name (Divide Name (ID)), unique serial number of the device (Serial Number), battery current status (Battery Life) and sensors assigned to each communication port. In the Communication terminal is possible to view all messages exchanged between the application and the equipment. It is also possible to define the data recording interval in the datalogger's internal memory (Measurement Interval);

- C - Datalogger Memory - Download of new data from datalogger's internal memory for the database in MS Excel files. It is possible to see the memory status, to clear the data logger internal memory and to open a new window for data correlation;

- D - Real Time Data - Shows weather conditions in real time with a minimum sample acquisition time of 2 seconds. The compass wind is updated in real time (depending on the user-defined acquisition time; $1 \mathrm{sec}, 10 \mathrm{sec}, \ldots, 1 \mathrm{~min}$ ), indicating wind direction. Enables the record of meteorological data in real time to the database (Weather Data Base);

- $\quad \mathbf{E}$ - Day Weather - Presents the weather conditions for the current day in two graphics. Also gives information about the maximum and minimum values achieved in the present day.

In addition to the main window, the user can open another window where a he can choose the variables that he wants to correlate, as presented in Fig. 5-(A). The variables that can be correlated were previously presented in Table 1.

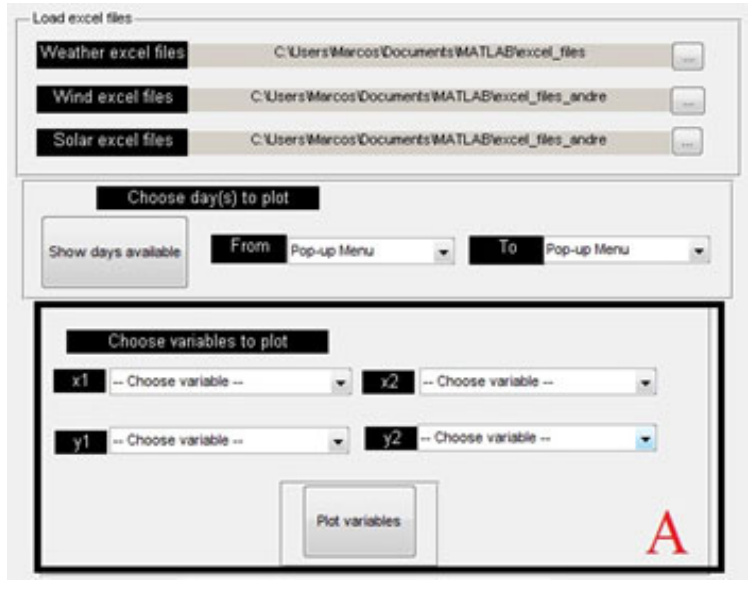

Fig. 5. Correlation window

In Fig. 5 window the user can create correlation graphics for the chosen variables, relating weather conditions and electrical parameters of the two renewable energy production systems. In addition to those variables, the total electrical energy produced 
and the power factor, for the selected time range, are shown. A new window (Fig. 6) presenting the obtained correlation graphics is generated. This window also offers the user the possibility of applying multiple filters to the correlation graphics. The maximum and minimum values of each curve are also displayed.

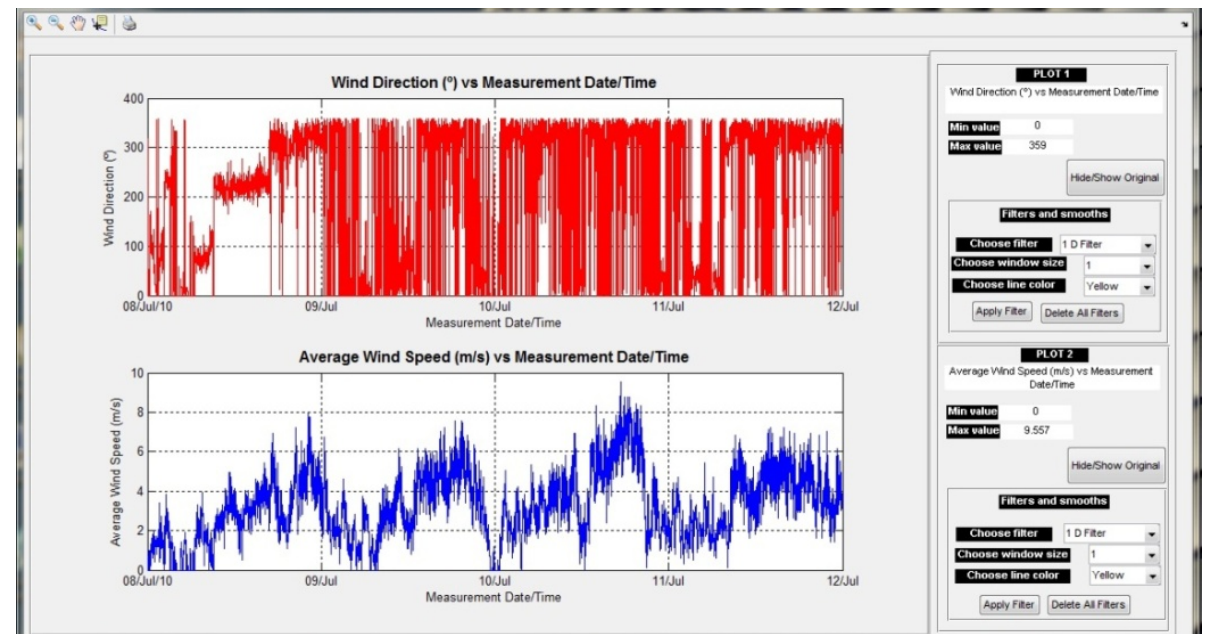

Fig. 6. Correlation window

\section{Experimental Results}

In this Section, experimental data obtained from the weather station and energy monitoring system, in the period of 23-29 of July 2010, is graphically presented. The weather station was set up acquisition sample intervals of one minute, resulting in 1440 readings per day. The energy monitoring system has acquisition sample intervals of 10 seconds, resulting in approximately 8000 readings per day. The wind generator is a $2 \mathrm{~kW}$ production system, and the PV has $460 \mathrm{Wp}$ installed.

\section{Correlation between weather conditions and wind generator production}

Figure 7 shows three graphics were it is possible to see the wind speed, wind power, wind generator electrical power and the system power coefficient $(\mathrm{Cp})$ over the period in analysis (Please note that the third graphic refers to the highlighted area in the second graphic). The highly oscillatory behaviour of the wind power and power coefficient are due to the sudden and unpredictable wind changes, which can be seen in the first graphic. The black line denotes average values of wind speed and $\mathrm{Cp}$. Wind power and generator electrical power have identical behaviour, which means that the energy produced follows the wind speed variation. As expected, wind power is higher than generator electrical power, denoting a typical $\mathrm{Cp}$ of 0.3 . The $\mathrm{Cp}$ is usually used as an indicator of the wind turbine efficiency and takes values between 0.3 and 0.4 , for common real systems. According to Betz's law, only less than 59\% (0.6) of the kinetic energy of wind can be converted into mechanical energy. 

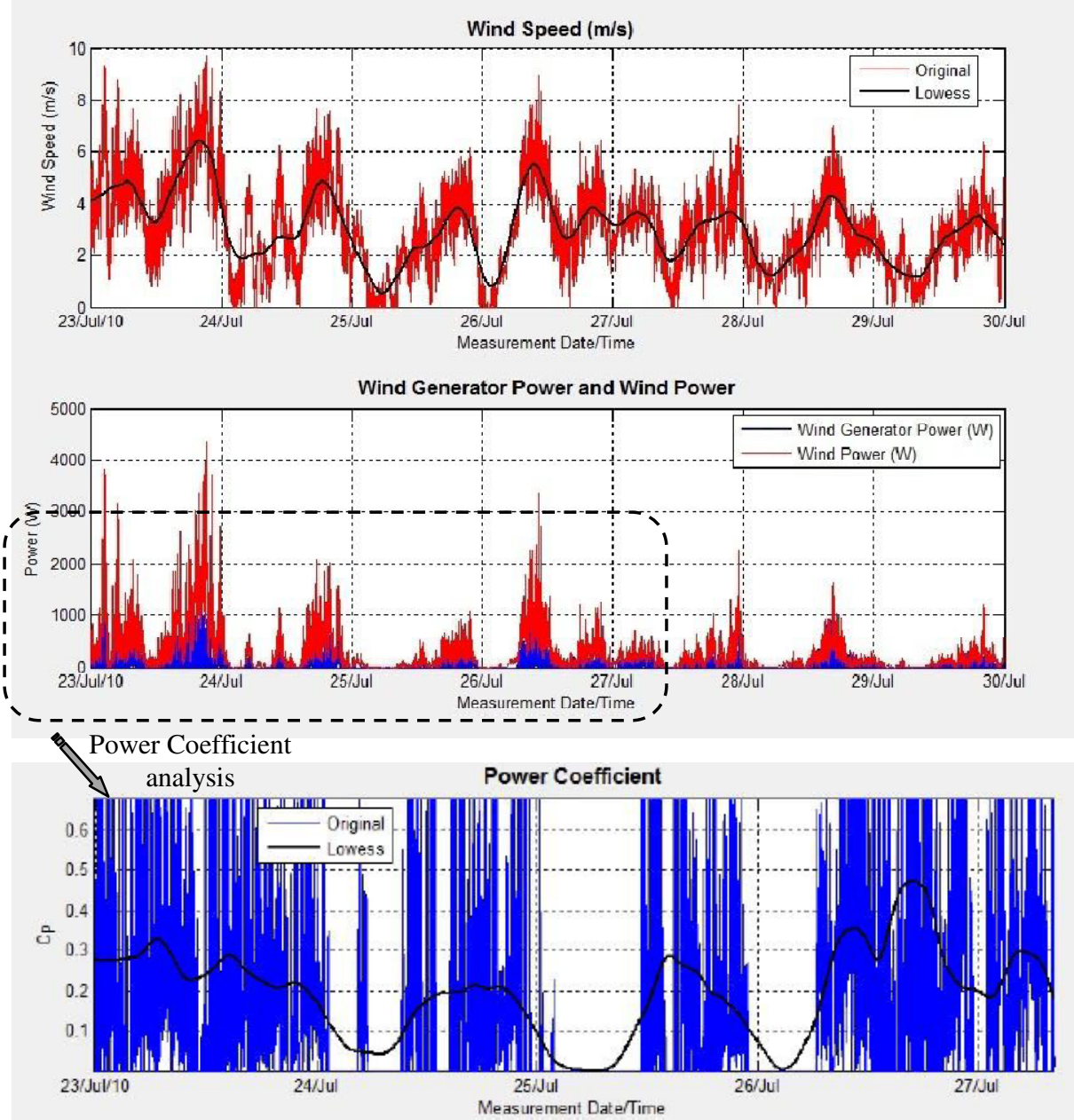

Fig. 7. Weather condition and wind generator energy production

\section{Correlation between weather conditions and PV production}

Fig. 8 shows the solar radiation, PV power and PV system efficiency. The electrical power generated by the PV system has, as expected, a similar behaviour as the solar radiation curve. The peak power reached is in concordance with the values of higher solar radiation measured on each day.

Another key point about this PV system is its efficiency. PV cells specified efficiency is obtained under Standard Test Conditions (STC), which means that the real PV efficiency largely depends on the place, and respective weather conditions, where the system is installed. For the system under analysis, the STC efficiency is about $7.2 \%$. However, as it is possible to see in Fig. 8, the real efficiency of the system is around 5\%. For PV panels the efficiency increases with solar radiation but decreases with the temperature of the panel. As expected, maximum efficiency occurred when 
maximum solar radiation was achieved. But, for maximum solar radiation, a high temperature value was also measured. High temperatures, over $25-27^{\circ} \mathrm{C}$, influences negatively the PV efficiency. This is the reason why, in Fig. 8, the efficiency of the system was slightly higher in point 2 than in 1, even with less radiation the temperature was lower in point 2.
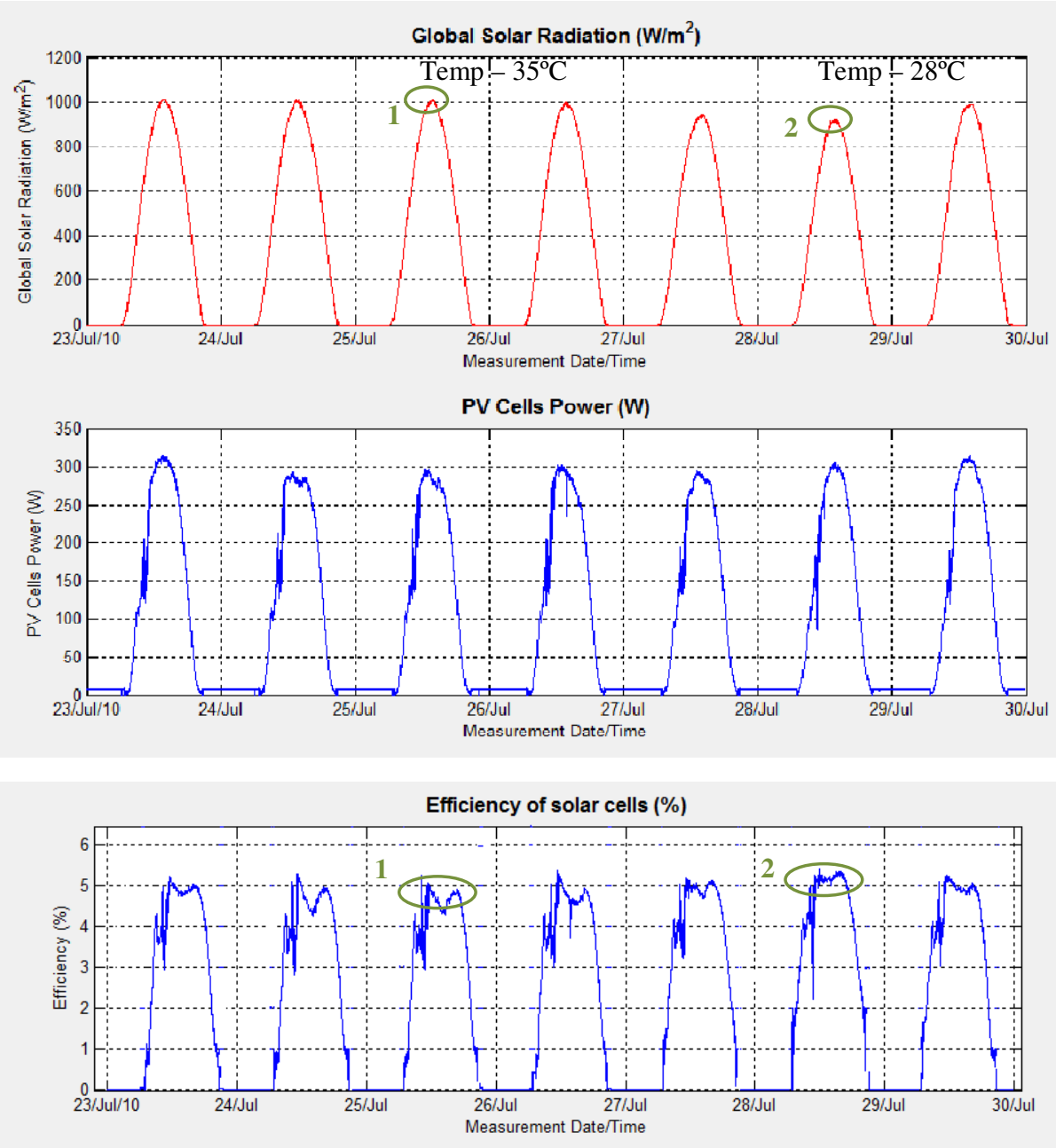

Fig. 8. Weather condition and PV system

\section{Conclusions}

This work has presented a system that integrates weather conditions information and energy parameters from renewable energy power generation system. The system incorporates an integrated analysis of the multiple variables involved, through an 
interactive graphical user interface. One of the advantages of an accessible and interactive user interface is the simple analysis of the renewable power systems generated electrical energy in relation to the existing weather conditions.

To validate the developed application, it was used data from a wind generator and photovoltaic panels, installed near to the weather station. The application developed in this work allows, in addition to the acquisition of weather and energy data, their monitoring and correlation through a simple and attractive graphical user interface, providing an easy interactivity with the user.

The implemented system can be used by institutions or companies related to renewable energy systems, meteorology, or even by the private user who is interested in monitoring the production of electricity from renewable energy systems installed in their homes.

Acknowledgments. This work was supported by FCT (CTS multiannual funding) through the PIDDAC Program funds.

\section{References}

1. Elhadidy, M., Shaahid, S.: Parametric Study of Hybrid (wind+solar+diesel) power generating Systems. Renew Energy 21(2), 129-139 (2000)

2. IEA: World Energy Outlook 2009. International Energy Agency Publications (2008)

3. Benghanem, M., Maafi, A.: Data Acquisition System for Photovoltaic Systems Performance Monitoring. IEEE Transactions on Instrumentation and Measurement 47, 30-33 (1998)

4. Mukaro, R., Carelse, X.F.: A Microcontroller-Based Data Acquisition System for Solar Radiation and Environmental Monitoring. IEEE Transactions on Instrumentation and Measurement 48, 1232-1238 (1999)

5. Forero, N., Hernández, J., Gordillo, G.: Development of a monitoring system for a PV solar plant. Energy Conversion and Management 47(15-16), 2329-2336 (2006)

6. Koutroulis, E., Kalaitzakis, K.: Development of an integrated data-acquisition system for renewable energy sources systems monitoring. Renewable Energy 28(1), 139-152 (2003)

7. Blaesser, G.: PV system measurements and monitoring: The European experience. Solar Energy Materials and Solar Cells 47(1-4), 167-176 (1997)

8. Jorge, A., Guerreiro, J., Pereira, P., Martins, J., Gomes, L.: Energy Consumption Monitoring System for Large Complexes. In: Camarinha-Matos, L.M., Pereira, P., Ribeiro, L. (eds.) DoCEIS 2010. IFIP AICT, vol. 314, pp. 22-24. Springer, Heidelberg (2010) 